\title{
Vision screening in preschoolers: the New South Wales Statewide Eyesight Preschooler Screening program
}

Stephanie J Blows MB BS, MPH(Hons), PhD Medical Epidemiologist

Elisabeth P Murphy MB BS(Hons) MPaed, FAFPHM Senior Clinical Advisor

Frank J Martin MB BS, FRANZCO, FRACS, Clinical Professor, Paediatric and Chlld Health and Ophthalmology

Robyn M Davies DipAppSci(Orth) GCertPubSecMgmt Senior Analyst

1 Child and Family Health, NSW Kids and Families, Sydney, NSW.

2 The Children's Hospital at Westmead Sydney, NSW.

Imurp@

doh.health.nsw.gov.au

MJA 2014; 200: 222-225 doi: 10.5694/mjal3.10594

Online first 24/02/14 ision assessment is an important component of preventive health in childhood. Prevalence estimates for visual disorders in Australian children suggest rates of about $2 \%$ for amblyopia (reduced visual acuity in one or both eyes with no pathological cause), ${ }^{1}$ up to $7.3 \%$ for strabismus, and between $1 \%$ and $14.7 \%$ for refractive error. ${ }^{2}$ Many of these disorders can only be diagnosed through a monocular visual acuity screen and cannot be identified by family history, vision surveillance or observation of the child's behaviour or appearance alone. Optimal treatment outcomes for childhood vision disorders are achieved with early detection and treatment, preferably before school entry. ${ }^{3}$ Failure to detect and treat vision disorders during childhood may lead to permanent loss of vision. ${ }^{4}$

There is evidence that a visual acuity test at about 4 years of age is best practice for preventing and treating vision disorders. ${ }^{2,5,6}$ Screening before school entry is ideal to optimise treatment outcomes ${ }^{1}$ and maximise compliance with treatment. ${ }^{6}$ All Australian states and territories offer some form of vision surveillance or screening to children: some recommend screening for all children before school entry, while others only screen children who are at risk or have a concern with their vision. ${ }^{6}$ However, child vision surveillance and screening typically rely on the child attending regular health checks, and systems that rely on parental vigilance are known to have variable participation rates. ${ }^{1,6}$

In New South Wales, vision surveillance is recommended as part of regular child health checks, as documented in the NSW Personal Health Record. From 2008, this was formalised as the Statewide Eyesight Preschooler Screening (StEPS) program, a free, universal vision screening program for 4-year-old children. Rather than relying on health check

\section{Abstract}

Objective: To evaluate screening and diagnostic outcomes of the New South Wales Statewide Eyesight Preschooler Screening (StEPS) program, a statefunded, universal vision screening program for 4-year-old children.

Design, setting and participants: A cross-sectional evaluation of the StEPS program, in which eligible 4-year-old children were offered a vision screen in local health districts in NSW, between 1 July 2010 and 30 June 2011.

Main outcome measures: Number and proportion of eligible children who were offered screening; accepted screening; were screened and scored a pass or were referred (routinely or urgently) for further vision assessment; and were referred for further assessment and required intervention.

Results: Of 91324 eligible 4-year-olds in NSW, 80328 (88.0\%) were offered screening, and 65834 ( $72.1 \%$ of the eligible population) were screened. Of the children who were screened, 3867 (5.9\%) scored less than 6/9-2 but better than $6 / 18$ in one or both eyes and were referred to their general practitioner or eye health professional for further vision assessment. A further 1425 children $(2.2 \%)$ scored $6 / 18$ or less in one or both eyes and were referred for high-priority assessment. In the two local health districts with the most complete follow-up data, 704 of 779 children (90.4\%) with routine referrals and 278 of 285 (97.5\%) with high-priority referrals required treatment or review at a later date.

Conclusions: The StEPS program has achieved a high screening participation rate in NSW. Many children have been diagnosed and received treatment for previously undetected serious vision disorders that may otherwise have been diagnosed too late for effective intervention.

attendance, the StEPS program actively identifies and targets all 4year-old children in NSW through preschools, childcare and other children's services. Children attending the service who are 5 years old and have not previously received a StEPS screen are also eligible for screening.

The program is administered by local health districts (LHDs) - eight in the Sydney metropolitan region and seven covering regional and rural NSW. Ideally, LHDs attempt to offer vision screening to $100 \%$ of 4 -year-old children but, as it may not be possible to reach all children, a minimum target of $90 \%$ has been set. ${ }^{7}$ Each LHD employs a StEPS Coordinator or assigns a staff member to oversee local implementation of the program. The coordinators are responsible for ensuring maximum program coverage and equity of access, and offer assistance to families to ensure that children identified through screening as having a possible vision disorder receive appropriate referral and diagnostic assessment. They also monitor and report on screening outcomes using data provided by LHDs.
Our aim was to evaluate the StEPS program to assess initial screening, referral and diagnosis rates for the target population.

\section{Methods}

We obtained de-identified data, collected between 1 July 2010 and 31 June 2011, from the LHD StEPS databases. Consent was sought from parents or carers for screening, and vision screening tests were conducted by StEPS vision screening staff who are trained and employed by each LHD. Data items collected on consent forms and at the time of screening were: demographic data; Indigenous status; personal and family history of vision disorders; vision screening results for each eye; result of screening (pass, borderline pass, type of referral); and other observations by the screener that were indications for referral, such as abnormal head posture or external eye abnormalities. In the event of a referral, data on the outcome or diagnosis after further investigation were also retrieved. Target population estimates for the study period were based 


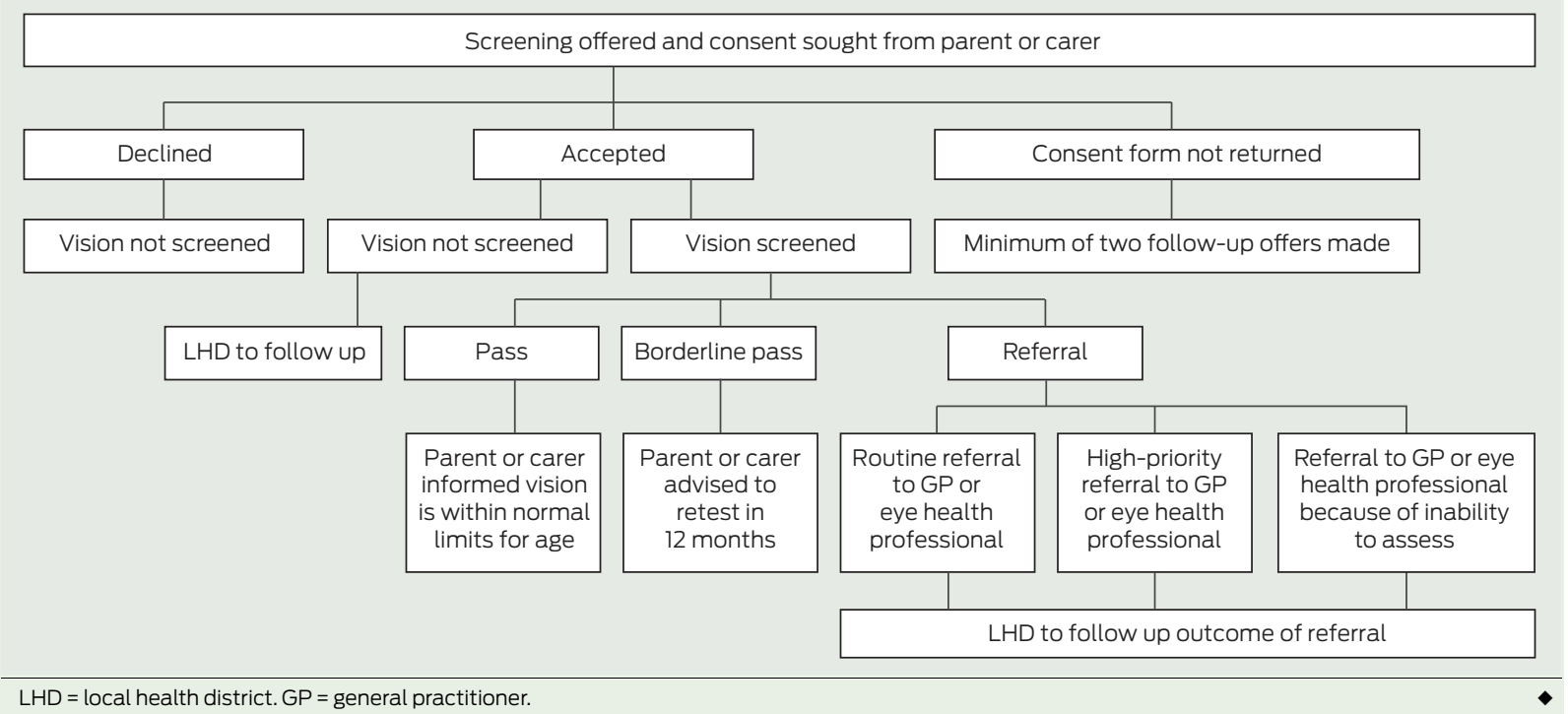

on population projections using 2006 Census data ${ }^{8}$ provided to us by the Statewide Services Development Branch of the NSW Ministry of Health in collaboration with the NSW Department of Planning. As this evaluation was an analysis of routinely collected program data for the monitoring and quality improvement of health service delivery, ethics approval was not required.

All screening and referral was done according to StEPS protocols. The StEPS referral pathway is shown in Box 1. If a consent form was not returned, a minimum of two followup screening offers were made. If a child was absent on the day of screening, parents or carers were asked to have their child's vision assessed through their child and family health centre or general practitioner. Children were screened using a $6 \mathrm{~m}$ linear chart (or $3 \mathrm{~m}$ chart where a distance of $6 \mathrm{~m}$ was not available). Children passed the screening test if their visual acuity was $6 / 9$ or above in each eye. A score of $6 / 9-1$ or $6 / 9-2$ in one or both eyes was regarded as a borderline pass, and parents or carers were advised to retest in 12 months. Children were referred to a GP or eye health professional for follow-up: if they scored less than 6/9-2 but better than 6/18 in one or both eyes; if there were obvious external eye abnormalities or the child's vision could not be assessed on the day of screening (eg, if the child was unwell or distracted); or if they scored $6 / 18$ or less in one or both eyes - this was regarded as a high-priority referral, and parents were advised to have the children urgently undergo further testing.

Completeness of data on diagnostic outcomes after referral from the StEPS screening program varied by LHD. Combined results from the two LHDs (which comprised one area health service at the time of the study) with the most complete data for the study period were analysed in more detail to enable better evaluation of outcomes.

\section{Results}

Of 91324 eligible 4-year-olds in NSW during the study period, 80328 $(88.0 \%)$ were offered screening. Of these, the parents or carers of 71081 children $(88.5 \%)$ accepted and provided consent for screening. Of those for whom consent was obtained, 65834 children $(72.1 \%$ of the eligible population) were screened. The remaining children for whom consent was received but who were not screened were absent on the day of screening. Of 74249 parents or carers who returned consent forms, 1600 $(2.2 \%)$ indicated they were declining because their child had already received a vision screen. Of children who were screened, 2568 (3.9\%) identified as being of Aboriginal and/ or Torres Strait Islander origin.
Outcomes of the testing were that 52870 children $(80.3 \%)$ passed the screening test, $6405(9.7 \%)$ received a borderline pass, and 6421 (9.8\%) were referred to their GP or eye health professional for further assessment. The group referred for further assessment comprised 3867 children (5.9\%) with routine referrals, 1425 (2.2\%) with high-priority referrals, and 1129 $(1.7 \%)$ who were unable to be assessed on the day of screening. Screening results were missing for 138 children $(0.2 \%)$, most likely due to inaccurate data entry.

The two LHDs (one metropolitan and one rural) with the most complete follow-up data for the study period achieved an average follow-up rate of $95.7 \%(1231 / 1286)$. These two LHDs are considered representative of metropolitan and rural LHDs in NSW, with an average referral rate of $9.9 \%$ of children (1286/12 977) screened. Box 2 shows the primary diagnoses for children followed up after screening in these two LHDs. Of 304 children with high-priority referrals, $28.0 \%$ were later diagnosed with amblyopia, $44.1 \%$ were prescribed glasses, and $5.6 \%$ were diagnosed with other vision disorders, including cataract, ptosis, glaucoma and nystagmus. Among 847 children with routine referrals after screening, 5.9\% were later diagnosed with amblyopia, $32.0 \%$ were prescribed glasses, and $14.8 \%$ were diagnosed with other vision disorders. Some of the children 
2 Primary diagnoses or other outcomes for preschoolers referred for further investigation after screening in the New South Wales Statewide Eyesight Preschooler Screening Program in two local health districts, July 2010 to June 2011

\begin{tabular}{lccc} 
Primary diagnosis or other outcome & $\begin{array}{c}\text { High-priority referrals } \\
(n=304)\end{array}$ & $\begin{array}{c}\text { Routine referrals } \\
(n=847)\end{array}$ & $\begin{array}{c}\text { Referred due to inability } \\
\text { to assess }(n=135)\end{array}$ \\
\hline Diagnoses & & & \\
Amblyopia & $76(25.0 \%)$ & $33(3.9 \%)$ & $1(0.7 \%)$ \\
$\quad$ Anisometropic amblyopia & $9(3.0 \%)$ & $17(2.0 \%)$ & $1(0.7 \%)$ \\
Strabismic amblyopia & $85(28.0 \%)$ & $50(5.9 \%)$ & $6(4.4 \%)$ \\
Total amblyopia & & & 0 \\
Prescribed glasses & $109(35.9 \%)$ & $242(28.6 \%)$ & $6(4.4 \%)$ \\
Refractive error & $25(8.2 \%)$ & $29(3.4 \%)$ & $5(3.7 \%)$ \\
Anisometropia & $134(44.1 \%)$ & $271(32.0 \%)$ & 0 \\
$\quad 17(5.6 \%)$ & $125(14.8 \%)$ & $12(8.9 \%)$ \\
Total prescribed glasses & 0 & $59(7.0 \%)$ & $18(13.3 \%)$ \\
Strabismus or squint & $505(59.6 \%)$ & $34(25.2 \%)$ \\
Total visual abnormality diagnoses & $236(77.6 \%)$ & & $2(1.5 \%)$ \\
Other outcomes & & $75(8.9 \%)$ & $42(31.1 \%)$ \\
No visual abnormality detected & $7(2.3 \%)$ & $197(23.3 \%)$ & $27(20.0 \%)$ \\
Review and monitoring indicated & $18(5.9 \%)$ & $2(0.2 \%)$ & $48(5.7 \%)$ \\
Already under the care of an eye health professional & $24(7.9 \%)$ & $20(2.4 \%)$ &
\end{tabular}

who were referred because they could not be assessed were also diagnosed with vision disorders: for example, $4.4 \%$ were prescribed glasses and $25.2 \%$ required monitoring and later review.

In addition to vision disorders, anecdotal evidence from the two LHDs indicated that some children who were referred because they could not be assessed had other diagnoses such as developmental delay.

Only a small proportion of children who were referred for further investigation after screening were found to have no visual abnormality (Box 2). Follow-up data were available for 285 of the 304 high-priority referrals (93.8\%) and, among these, there were only seven children in whom no abnormality was found. Therefore, 278 of 285 children $(97.5 \%)$ for whom follow-up data were available required either treatment or review at a later date. For routine referrals, 704 of 779 children $(90.4 \%)$ who received a follow-up assessment required either treatment for a vision problem or review at a later date.

\section{Discussion}

Data from this evaluation indicate that the StEPS program is close to achieving its aim of offering vision screening to at least $90 \%$ of 4 -year-old children in NSW. The high proportion of parents being offered and consenting to screening for their children indicates a high level of acceptance of screening in this population. Our results suggest the program can also be successfully offered to specific groups of children, such as those from Aboriginal and Torres Strait Islander backgrounds. We found that the proportion of screened children who identified as Aboriginal and/or Torres Strait Islander $(3.9 \%)$ was similar to the estimated percentage of all 4year-old Aboriginal and/or Torres Strait Islander children in NSW. ${ }^{9}$

Although many jurisdictions worldwide screen children's vision at or after the time of school entry, only a few, such as Sweden and parts of Canada, undertake universal visual acuity screening before school entry. ${ }^{5}$ Follow-up data indicate that preschool screening programs reduce the incidence of vision disorders, ${ }^{10}$ with one cohort study finding a $45 \%$ reduction in the prevalence of amblyopia among 7-year-olds who received preschool screening, compared with those who did not. ${ }^{11}$ Although reviews of the literature have found a lack of evidence of effectiveness for preschool vision screening programs, due to a lack of well designed randomised controlled trials, ${ }^{2,12,13}$ expert opinion suggests that the value of early intervention is sufficient that screening programs should be implemented on the strength of existing evidence. $^{1,6}$

Our data support the likelihood of considerable benefits for children receiving a preschool vision screen. For the 6421 children we identified as requiring further assessment after screening, early detection and treatment may have prevented loss of vision from conditions such as anisometropic amblyopia, ${ }^{4}$ which was diagnosed in $25 \%$ of children with high-priority referrals. This potentially serious disorder is not detectable by vision surveillance alone, and failure to treat it at a young age may lead to permanent loss of vision. ${ }^{14}$ Treatments for amblyopia, such as patching, are not only more effective during the preschool years, they are also more appropriate, as patching at school may lead to bullying. ${ }^{6}$ A significant proportion of children referred for investigation in our study were also prescribed glasses before entering school.

Before the implementation of the StEPS program, vision assessment in preschool children in NSW relied on voluntary attendance at the 4-yearold child health check, for which there is some evidence that attendance is poor, particularly among the most socioeconomically disadvantaged 
groups. ${ }^{15}$ We found that only $2 \%$ of parents declined consent because their child had previously had a vision screen. In addition, vision surveillance conducted during health checks does not necessarily include a monocular visual acuity screen. ${ }^{6}$ Thus, without universal preschool screening, many children with vision problems are likely to remain undiagnosed, and their vision uncorrected, into their school years. Preschool screening is preferable to school screening, as the critical window for intervention may otherwise be lost. ${ }^{6}$

The StEPS program aims to make the best possible treatment readily available to the screened population by offering assistance to families to ensure that children receive appropriate diagnostic assessment and referral. The StEPS Coordinator may provide a referral either to a GP, who can then refer to an eye health professional as appropriate, or directly to one of the dedicated tertiary paediatric ophthalmic outpatient clinics that have been established in NSW for children referred via the program. Maximising positive treatment outcomes after early diagnosis is an important feature of screening programs. ${ }^{16}$ We found that only $1.7 \%$ of children were unable to be assessed on the day of screening, which demonstrates the appropriateness of this screening in our target group. Use of this test for screening allows more costly, invasive and time-consuming investigations to be reserved for those identified to be at higher risk. Our data indicate that among children referred from the StEPS program for further investigation, only a small proportion (2.3\% of high-priority and $8.9 \%$ of routine referrals) had no abnormality diagnosed, suggesting this screening test is accurate and that the referral criteria in the StEPS program are appropriate.

There are three important limitations of this study. First, because children who tested negative for vision disorders during screening were not followed up as part of StEPS to deter- mine their true disease status, we were not able to calculate sensitivity or specificity of the screening test. A second limitation is incompleteness of our data on vision disorders that are diagnosed after referral from the StEPS program. Although each LHD is required to follow up visual outcomes for children who are referred for further assessment, this has proven difficult in many districts because of the diversity of services that provide follow-up. The StEPS policy directive is currently being revised to ensure consistency of diagnostic outcome reporting from all LHDs, and accuracy and completeness of outcome data have significantly improved since mid 2012. Future evaluation studies will benefit from this more complete outcome data measured over a longer period. Finally, this evaluation could not measure the cost-benefit ratio of screening, in financial terms and with respect to the costs, risks and benefits for individual children. ${ }^{16}$ Initial funding of the program amounted to about \$14 million over 4 years for both the StEPS program and the paediatric ophthalmic outpatient clinics. Our evaluation focused only on screening and diagnostic outcomes, but the cost-effectiveness of the StEPS program is an important area for future research. Although the program would ideally exist as part of a universal comprehensive health screening program before school entry, vision screening is considered of sufficient importance that the StEPS program should be conducted in isolation in the meantime.

In conclusion, the StEPS program is conducting visual acuity testing in a high proportion of 4-year-olds in NSW. Prompt referral and optimal treatment of common visual disorders may prevent potentially permanent loss of vision in a significant number of children. The StEPS model may be useful for other jurisdictions in Australia and internationally considering implementation of similar programs.
Acknowledgements: We wish to acknowledge the StEPS Coordinators in each LHD for their contribution to the StEPS program and their role in data collection for this study.

Competing interests: No relevant disclosures.

Received 4 May 2013, accepted 13 Nov 2013.

1 Pai AS, Rose KA, Leone JF, et al. Amblyopia prevalence and risk factors in Australian preschool children. Ophthalmology 2012; 119: 138-144.

2 Mathers M, Keyes M, Wright M. A review of the evidence on the effectiveness of children's vision screening. Child Care Health Dev 2010; 36: 756-780.

3 Royal Australian and New Zealand College of Ophthalmologists. "Lazy eye" amblyopia. http:// www.ranzco.edu/index.php/patients/eyeconditions/qlazyq-eye-amblyopia (accessed Jul 2012).

4 Doshi NR, Rodriguez ML. Amblyopia. Am Fam Physician 2007; 75: 361-367.

5 Mema SC, McIntyre L, Musto R. Childhood vision screening in Canada: public health evidence and practice. Can J Public Health 2012; 103: 40-45.

6 Morcos A, Wright M. National Children's Vision Screening Project: final report. Melbourne: Murdoch Childrens Research Institute, 2009. http://www.rch.org.au/uploadedFiles/Main/ Content/ccch/Vision_Screening_Final_Report_ May_2009.pdf (accessed May 2012).

7 Ministry of Health, NSW. Statewide Eyesight Preschooler Screening (StEPS) Program. Policy directive PD2012_001. Sydney: NSW Health, 2012. http://www.health.nsw.gov.au/policies/pd/ 2012/pdf/PD2012_001.pdf (accessed May 2012).

8 Australian Bureau of Statistics. 2006 Census data. http://abs.gov.au/websitedbs/ censushome.nsf/home/historicaldata2006 (accessed Nov 2011).

9 New South Wales Commission for Children and Young People. A picture of NSW children [website]. http://www.picture.kids.nsw.gov.au (accessed Sep 2012).

10 Lennerstrand G, Jakobsson P, Kvarnstrom G. Screening for ocular dysfunction in children: approaching a common program. Acta Ophthalmol Scand Suppl 1995; (214): 26-38.

11 Williams C, Northstone K, Harrad RA, et al. Amblyopia treatment outcomes after preschool screening v school entry screening: observational data from a prospective cohort study. $\mathrm{Br} J$ Ophthalmol 2003; 87: 988-993.

12 Schmucker C, Grosselfinger R, Riemsma R, et al. Effectiveness of screening preschool children for amblyopia: a systematic review. BMC Ophthalmol 2009; 9: 3.

13 Powell C, Hatt SR. Vision screening for amblyopia in childhood. Cochrane Database Syst Rev 2009; (3): CD005020. doi: 10.1002/14651858.CD 005020.pub3.

14 Donahue SP. The relationship between anisometropia, patient age, and the development of amblyopia. Trans Am Ophthalmol Soc 2005; 103: 313-336.

15 Centre for Epidemiology and Evidence. New South Wales Child Health Survey: 2009-2010 summary report. Sydney: NSW Ministry of Health, 2012. http://www.health.nsw.gov.au/surveys/ child/Pages/hsc_0910.aspx (accessed Jul 2012).

16 Birbeck GL. The benefits of screening must outweigh the risks and costs [comment]. West J Med 2000; 172: 308-309. 